\title{
Détermination de la contamination par l'Aflatoxine B1 de la pâte d'arachide consommée par la population en Côte d'Ivoire : intérêt de la Chromatographie sur Couche Mince
}

\author{
Aissata DIAKITE ${ }^{1,3^{*}}$, Marc GOULI BI IRIE ${ }^{2}$, Dakmak Kouassi N'DRI ${ }^{2}$ et \\ Jacques Aboua $\mathrm{YAPO}^{2}$ \\ ${ }^{1}$ Laboratoire de Toxicologie et Hygiène Agro-Industrielle (LTHAI), UFR Sciences Pharmaceutiques et \\ Biologiques, Université Félix Houphouët-Boigny, Abidjan, BP V 34, Côte d'Ivoire \\ ${ }^{2}$ Laboratoire de Chimie-Physique, UFR Sciences des Structures de la Matière et Technologie, Université Félix \\ Houphouët-Boigny, Abidjan, BP V34, Côte d'Ivoire \\ ${ }^{3}$ Laboratoire National de la Santé Publique, Laboratoire de Toxicologie. 52, Boulevard de Marseille. 18 BP \\ 2403 Abidjan 18, Côte d'Ivoire \\ "Auteur correspondant; E-mail: a_diak@hotmail.fr / BP V34 Abidjan, Côte d'Ivoire.Tél: +22547212121
}

\section{RÉSUMÉ}

Les arachides sont très vulnérables à la contamination par les Aflatoxines B1 (AFB1) reconnues pour leurs propriétés hautement toxiques, mutagènes, cancérigènes, tératogéniques et hépatotoxiques. En Côte d'Ivoire, la pâte d'arachide est largement consommée, en particulier par les enfants, en raison de ses qualités nutritionnelles exceptionnelles. L'objectif de notre étude était, d'une part, de déterminer le seuil de détection de l'AFB1 dans la pâte d'arachide par la méthode de Chromatographie sur Couche Mince (CCM). D'autre part, de déterminer par CCM si les niveaux de contamination naturelle par l'AFB1 des pâtes d'arachide vendues sur les marchés d'Abidjan étaient supérieurs aux limites maximales de résidus autorisées. Ainsi, des échantillons de pâte d'arachide (blanc-matrice) ont été contaminés expérimentalement avec des quantités connues et décroissantes d'un standard d'AFB1. Après extraction, ces échantillons ont été analysés par CCM afin d'identifier la concentration minimale en AFB1 donnant une réponse positive. Douze échantillons collectés sur les marchés d'Abobo, Adjamé et Yopougon ont été analysés selon la même méthode (CCM). Nos résultats démontrent que la méthode d'analyse mise en œuvre permet de détecter des niveaux de contamination par l'AFB1 de l'ordre de $2 \mu \mathrm{g} / \mathrm{kg}$. Ce niveau de concentration correspond à la Limite Maximale de Résidu (LMR) autorisée par 1'Union Européenne. L'analyse de différents échantillons de pâte d'arachide prélevés sur 3 marchés populaires d'Abidjan n'a pas révélé de contamination significative des échantillons testés.

(C) 2017 International Formulae Group. All rights reserved.

Mots clés : AFB1, pâte d'arachide, CCM, limite de détection, contrôle sanitaire.

\section{Detection and control of Aflatoxin B1 occurrence in peanut butter produced in Côte d'Ivoire: relevance of Thin Layer Chromatography}

\begin{abstract}
Peanuts are very sensitive to Aflatoxins B1 (AFB1) which are reputed to be highly carcinogenic, teratogenic and hepatotoxic. In Côte d'Ivoire, peanut butter is greatly appreciated by consumers and especially
\end{abstract}


by children for its exceptional nutritional value. The first aim of our study was to assess the sensibility of Thin Layer Chromatography (TLC) for the detection of AFB1 in peanut butter. Then, the analytical method was applied to peanut butter samples collected on 3 markets in Abidjan in order to verify if the contamination by AFB1 was within permissible limits. To this end, blank samples of peanut butter were spiked with known and decreasing concentrations of AFB1 standard. After extraction, samples were analyzed by TLC and the lower concentration that gave a positive response was identified as the Limit of Detection. Twelve samples from Abobo, Adjamé and Yopougon markets were analyzed by the same method. We demonstrated that the Limit of Detection of AFB1 contamination in peanut butter by TLC was $2 \mu \mathrm{g} / \mathrm{Kg}$. This level of contamination corresponds to the Maximum Residue Limit (MRL) established by European Union for AFB1 in peanuts. We did not found any contamination beyond the authorized MRL in all peanuts butter samples collected on the markets in Abidjan.

(C) 2017 International Formulae Group. All rights reserved.

Keywords: AFB1, peanuts butter, Thin Layer Chromatography, detection limit, food control.

\section{INTRODUCTION}

Les Aflatoxines (AF) sont des mycotoxines qui se développent sur une large variété de denrées alimentaires et particulièrement sur les arachides. Ces mycotoxines sont élaborées à partir de champignons microscopiques ou moisissures appartenant principalement aux genres Aspergillus et Penicillium qui prolifèrent surtout dans les régions chaudes et humides comme sous nos tropiques (Wacoo, 2014). En effet, nos conditions climatiques favorisent la production de moisissures aflatoxinogènes au cours du stockage post-récolte des arachides.

Il existe plusieurs structures d'aflatoxines dont les quatre majeures sont AFB1, AFB2, AFG1 et AFG2 qui contaminent les produits agricoles et posent des problèmes de santé humaine, animale et économique. En effet, ces Aflatoxines sont reconnues pour leurs propriétés hautement toxiques, mutagènes, cancérigènes, tératogéniques et hépatotoxiques. Chez les sujets porteurs de l'Hépatite $B$ ou de l'Hépatite $\mathrm{C}$, le risque de développer un cancer $\mathrm{du}$ foie est extrêmement élevé lorsqu'ils sont exposés aux Aflatoxines (Costanzo, 2015). Parmi les Aflatoxines, la forme la plus toxique est l'AFB1 (Figure 1). Par ailleurs, l'AFB1 a été classée par l'Agence Internationale pour la Recherche sur le Cancer (IARC) comme cancérigène humain du groupe 1 (IARC, 2002).

En raison de ces risques sur la santé des populations, les Aflatoxines font l'objet d'une réglementation très stricte dans de nombreux pays. Sangare-Tigori et collaborateurs (2006) ont évalué la contamination de certaines céréales et des arachides vendues sur différents marchés locaux en Côte d'Ivoire. Les auteurs rapportent des taux de contamination moyens de l'arachide par l'AFB1 de $4,8 \mu \mathrm{g} / \mathrm{kg}$. Il convient, cependant, de noter qu'en Côte d'Ivoire l'arachide est davantage consommée sous la forme de pâte d'arachide, particulièrement chez les enfants en raison de ses qualités gustatives et nutritionnelles exceptionnelles.

Cette pâte d'arachide est obtenue par un procédé de fabrication qui inclut une phase de rôtissage de l'arachide à des températures variables selon la recette de fabrication (Knoden, 2003). Parallèlement, certaines études ont montré que les Aflatoxines sont relativement stables et ne sont pas complètement détruites par la chaleur (Betina, 1989). Malheureusement, nous ne disposons pas de données sur le niveau de contamination par les Aflatoxines des pâtes d'arachide commercialisées en Côte d'Ivoire. À l'heure actuelle, il existe des méthodes très sensibles permettant de déterminer les teneurs en Aflatoxines dans les aliments contaminés (HPLC-FLD ou HPLC-MS/MS). Toutefois, ces dernières sont également très sophistiquées et coûteuses pour des analyses dans un pays à revenus faibles comme la Côte d'ivoire. Le but de notre étude est de valider une méthode simple, fiable et peu coûteuse de 


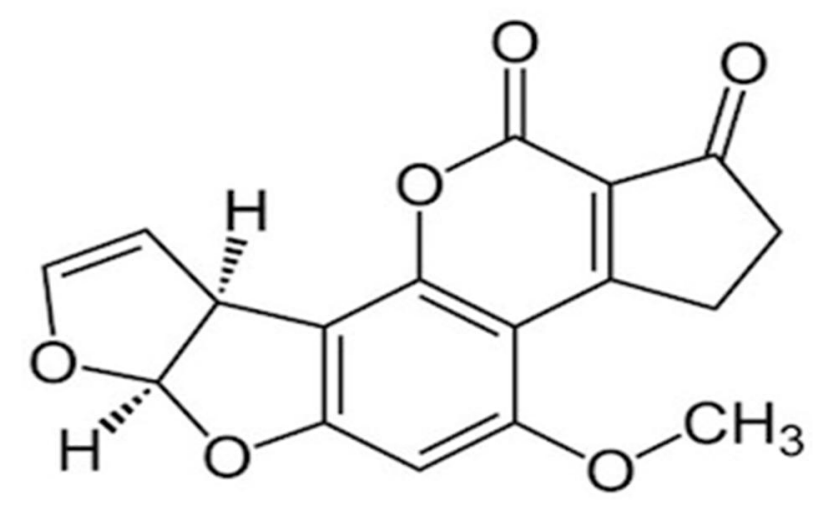

Figure 1 : Structure chimique de l'Aflatoxine B1 (AFB1); PM=213,2 (IARC, 2002).

détection des Aflatoxines dans la pâte d'arachide consommée par la population en Côte d'Ivoire.

Plus spécifiquement, les objectifs de notre étude étaient de :

1) Déterminer la Limite de Détection (LD) de l'AFB1 dans des échantillons de pâte d'arachide par Chromatographie sur Couche Mince (CCM) ;

2) Évaluer la contamination naturelle par AFB1 de plusieurs échantillons de pâte d'arachide prélevés sur le marché local par CCM.

\section{MATÉRIEL ET MÉTHODES}

Détermination de la limite de détection de l'AFB1 dans la pâte d'arachide

Afin de déterminer la Limite de Détection (LD) de l'AFB1 dans un échantillon de pâte d'arachide fabriquée en Côte d'Ivoire, nous avons utilisé un échantillon de pâte d'arachide non contaminé, préalablement analysé par HPLC-FLD. Cet échantillon qui représente le blanc-matrice a été, ensuite, contaminé par des quantités connues et décroissantes d'AFB1 afin d'obtenir les concentrations finales de $20 \mu \mathrm{g} / \mathrm{kg}, 10 \mu \mathrm{g} / \mathrm{kg}$,

$5 \mu \mathrm{g} / \mathrm{kg}$ et $2 \mu \mathrm{g} / \mathrm{kg}$. La contamination a été réalisée en triplicata pour chaque niveau de concentration, à l'aide d'un standard d'AFB1 (TRILOGY Analytical Laboratory, lot : $\quad 140115-022, \quad 25.0 \quad \mu \mathrm{g} / \mathrm{ml}$ dans Acétonitrile) selon le modèle mathématique de contamination suivant : $\quad \mathbf{Q}_{\mathbf{p}}=\left(\mathbf{P E} . \mathbf{C}_{\mathrm{fc}}\right)$ / $\mathbf{C}_{\text {STD }}$

$\mathbf{Q}_{\mathbf{p}} \quad:$ quantité du standard AFB1 à prélever (en $\mu \mathrm{L})$

PE : prise d'essai (en $\mathrm{kg}$ )

$\mathbf{C}_{\mathrm{fc}}$ : concentration finale du blancmatrice (en $\mu \mathrm{g} / \mathrm{kg}$ )

$\mathbf{C}_{\text {STD }}$ : concentration du standard $\mathrm{AFB} 1=25 \mu \mathrm{g} / \mathrm{mL}$

Ces échantillons ainsi contaminés ont été par la suite analysés par Chromatographie sur Couche Mince (CCM) selon le protocole décrit au point 3, afin de déterminer la concentration minimale pouvant être détectée par notre méthode.

\section{Échantillonnage}

Notre second objectif était d'évaluer le niveau de contamination des pâtes d'arachide fabriquées et commercialisées en Côte d'Ivoire. Pour cela, nous avons réalisé un échantillonnage du 12 avril au 12 mai 2016, auprès des vendeuses les plus sollicitées dans les marchés des trois quartiers les plus populaires d'Abidjan: Abobo (1,030,658 habitants), Adjamé (372,978 habitants) et Yopougon (1,071,000 habitants) (INS, RGPH 2014). Au total, 12 échantillons ont été collectés auprès des vendeuses de pâte d'arachide des différents marchés. Les échantillons étaient conditionnés dans des pots en plastique, acheminés au Laboratoire National de la Santé Publique, dans une 
glacière et conservés au réfrigérateur entre 2 $5^{\circ} \mathrm{C}$ avant d'être analysés par CCM.

\section{Préparation, extraction et purification des échantillons}

La méthode de préparation des échantillons appliquée dans notre expérimentation est celle de Boutibonnes (1969). Le mode opératoire comporte quatre étapes :

\section{- Extraction}

Vingt grammes de pâte d'arachide (échantillon contaminé ou échantillon du marché) sont introduits dans un erlenmeyer de $250 \mathrm{~mL}$ et additionnés de $100 \mathrm{~mL}$ d'un mélange méthanol : eau $(80: 20)$. Après 15 minutes d'agitation magnétique et filtration sur papier Whatman no 6 on obtient le Filtrat 1.

\section{- Déprotéinisation}

La précipitation des protéines s'est faite par ajout de $45 \mathrm{~mL}$ d'un mélange acide phosphotungstique $(5 \mathrm{~g})+$ sulfate de zinc $(15 \mathrm{~g})+$ eau distillée (11) à $50 \mathrm{~mL}$ du Filtrat 1 . La filtration sur papier Whatman no 6 aboutit au Filtrat 2.

\section{- Délipidation}

L'élimination des lipides du Filtrat 2 est obtenue par extraction en ampoule à décanter avec $3 \times 10 \mathrm{~mL}$ d'éther de pétrole. On obtient ainsi l'extrait aqueux 1 (Extrait 1)

\section{- $\quad$ Extraction de l'Aflatoxine B1}

A partir de l'extrait 1, les aflatoxines sont extraites avec 3 fois $10 \mathrm{ml}$ de chloroforme dans une ampoule à décanter. L'extrait chloroformique était recueilli dans une capsule et concentré par évaporation à un volume d'environ $0,5 \mathrm{~mL}$ (Extrait 2).

\section{Détection et évaluation semi-quantitative de l'Aflatoxine B1 \\ - Méthodes}

Afin de mettre en évidence l'Aflatoxine B1 dans la pâte d'arachide, nous avons utilisé des plaques de Chromatographie sur Couche Mince (CCM) en aluminium recouverte d'une phase stationnaire en gel de silice G60 de $0,25 \mathrm{~mm}$ d'épaisseur et de dimensions $10 \mathrm{~cm} \times 20 \mathrm{~cm}$ et $20 \mathrm{~cm} \times 20 \mathrm{~cm}$.
Pour chaque Extrait 2 obtenu, après purification, $5 \mu \mathrm{L}$ était déposés sur la plaque en présence d'un volume égal de standard AFB1 pour les pâtes d'arachide contaminées et les pâtes d'arachide collectées sur les différents marchés.

La plaque était éluée par un système binaire de solvants Chloroforme: acétone $(90 / 10 ; \mathrm{v} / \mathrm{v})$ puis les spots correspondants aux Aflatoxines étaient révélés en chambre noire sous un rayonnement UV produit par une lampe à $\lambda=365 \mathrm{~nm}$.

\section{- $\quad$ Analyses des résultats}

À la fin de la migration des composés par CCM, le rapport frontal (Rf) était calculé pour chaque échantillon. Le Rf représente le rapport entre la distance parcourue par la substance étudiée et la distance parcourue par le solvant.

Pour les échantillons contaminés, l'exposition aux UV à $365 \mathrm{~nm}$ pour AFB1 sur la plaque devait révéler une fluorescence bleue avec un $R f$ sensiblement égal à 0,56 (Abdellah, 2004). En revanche, les échantillons contaminés dont la concentration en AFB1 était inférieure au seuil de détection de notre méthode ne présentaient pas de fluorescence bleue. Pour les échantillons collectés sur les marchés locaux, les critères de positivité appliqués étaient exactement les mêmes.

\section{RÉSULTATS}

Limite de détection de l'AFB1 dans la pâte d'arachide

L'analyse des chromatogrammes obtenus par CCM des extraits de pâtes d'arachide contaminées expérimentalement a montré des spots fluorescents bleus avec un Rf $\sim 0,56$ pour tous les niveaux de contamination $(20 \mu \mathrm{g} / \mathrm{kg}, 10 \mu \mathrm{g} / \mathrm{kg}, 5 \mu \mathrm{g} / \mathrm{kg}$ et $2 \mu \mathrm{g} / \mathrm{kg})$. La fluorescence bleue observée est caractéristique de celle de l'AFB1 sous lumière UV (365 nm) et était comparable au témoin obtenu par dépôt direct et sans extraction préalable d'un standard pur d'AFB1. Ce Rf de 0,56 correspondant à la migration de l'AFB1 sur la phase stationnaire de la plaque de CCM, a été obtenu pour les quatre niveaux de contamination analysés en triplicata et est spécifique de l'AFB1 selon 
plusieurs auteurs (INRS, 2009; Abdellah, 2004). La comparaison de la fluorescence des échantillons contaminés et des $\mathrm{Rf}$ par rapport à ceux du témoin d'AFB1 pur permet de valider la positivité de nos résultats.

L'intensité de la fluorescence observée était directement proportionnelle au niveau de contamination de la pâte d'arachide par l'AFB1 (Figure 2). En effet, à l'analyse sous la lampe UV, plus la concentration en AFB1 dans l'extrait de pâte d'arachide augmentait, plus l'intensité de la fluorescence bleue était importante. Cette fluorescence bleue caractéristique de l'AFB1 et qui migre avec un Rf de 0,56 apparait dès le premier niveau de contamination, c'est-à-dire $2 \mu \mathrm{g} / \mathrm{kg}$, et s'intensifie au-delà. Des témoins d'Aflatoxines AFB2 (bleue, $\mathrm{Rf} \sim 0,53$ ), AFG1 (verte, Rf 0,51 ) et $\mathrm{AFG} 2$ (verte, $\mathrm{Rf} \sim 0,46$ ) ont été réalisés sur la même plaque afin de démontrer et confirmer que la tâche fluorescente bleue $(\mathrm{Rf} \sim$ $0,56)$ pour les 4 niveaux de contamination correspondait bien à l'AFB1 qui faisait l'objet de notre étude.

Nos résultats confirment que les méthodes d'extraction et d'identification mises en œuvre permettent de mettre en évidence des contaminations en AFB1 dans la pâte d'arachide à partir d'un seuil de $2 \mu \mathrm{g} / \mathrm{kg}$.

\section{Évaluation de la contamination des pâtes} d'arachide vendues sur le marché

$\mathrm{Au}$ total 12 échantillons de pâte d'arachide prélevés sur 3 marchés d'Abidjan ont été analysé en duplicata par la méthode décrite précédemment. Le mode d'échantillonnage et les caractéristiques organoleptiques des échantillons sont décrits dans le Tableau 1. Les échantillons collectés présentaient tous une odeur caractéristique et marquée d'arachide grillée. Cependant, la couleur et la consistance étaient différentes en fonction des vendeuses. Huit prélèvements présentaient une teinte marron foncé suggérant un rôtissage plus poussé des arachides alors que quatre prélèvements étaient de teinte beaucoup plus claire avec une texture plus lisse. L'analyse de ces échantillons par la méthode CCM dont nous avons au préalable pu déterminer la limite de détection de l'AFB1 dans la pâte d'arachide, n'a mis en évidence aucune tâche fluorescente bleue avec un $\mathrm{Rf} \sim 0,56$ après lecture à la lumière ultraviolet $(\lambda=365 \mathrm{~nm})$. Nous pouvons en déduire que les pâtes d'arachide prélevés sur le marché local à Abidjan ne présentaient pas de traces de contamination par les Aflatoxines B1 au-delà de $2 \mu \mathrm{g} / \mathrm{kg}$ qui représente la limite de détection de la méthode appliquée. Cette innocuité des échantillons testés se traduisait par l'absence totale de fluorescence bleue sur la plaque de CCM en comparaison avec le témoin AFB1.

Tableau 1 : Caractéristiques des échantillons de pâte d'arachides analysés.

\begin{tabular}{lcccccc}
\hline Marché & $\begin{array}{c}\text { Date de } \\
\text { prélèvement }\end{array}$ & $\begin{array}{c}\text { Nombre de } \\
\text { prélèvements }\end{array}$ & $\begin{array}{c}\text { Numéros } \\
\text { des } \\
\text { échantillons }\end{array}$ & $\begin{array}{c}\text { Quantités } \\
\text { prélevées } \\
\text { (gramme) }\end{array}$ & $\begin{array}{c}\text { Mode de } \\
\text { prélèvement }\end{array}$ & $\begin{array}{c}\text { Couleur de } \\
\text { l'échantillon }\end{array}$ \\
\hline Yopougon & $12 / 04 / 2016$ & 4 & $1,2,3,4$ & 300 à 500 & $\begin{array}{c}1 \text { par } \\
\text { vendeuse }\end{array}$ & $\begin{array}{c}\text { Marron }(1,2,4) \\
\text { Brun clair }(3)\end{array}$ \\
Adjamé & $26 / 04 / 2016$ & 4 & $5,6,7,8$ & 300 à 500 & $\begin{array}{c}1 \text { par } \\
\text { vendeuse }\end{array}$ & $\begin{array}{c}\text { Marron }(5,7,8) \\
\text { Brun clair }(6)\end{array}$ \\
Abobo & $12 / 05 / 2016$ & 4 & $9,10,11,12$ & 300 à 500 & $\begin{array}{c}1 \text { par } \\
\text { vendeuse }\end{array}$ & $\begin{array}{c}\text { Marron }(9,11) \\
\text { Brun clair } \\
(10,12)\end{array}$ \\
\hline
\end{tabular}




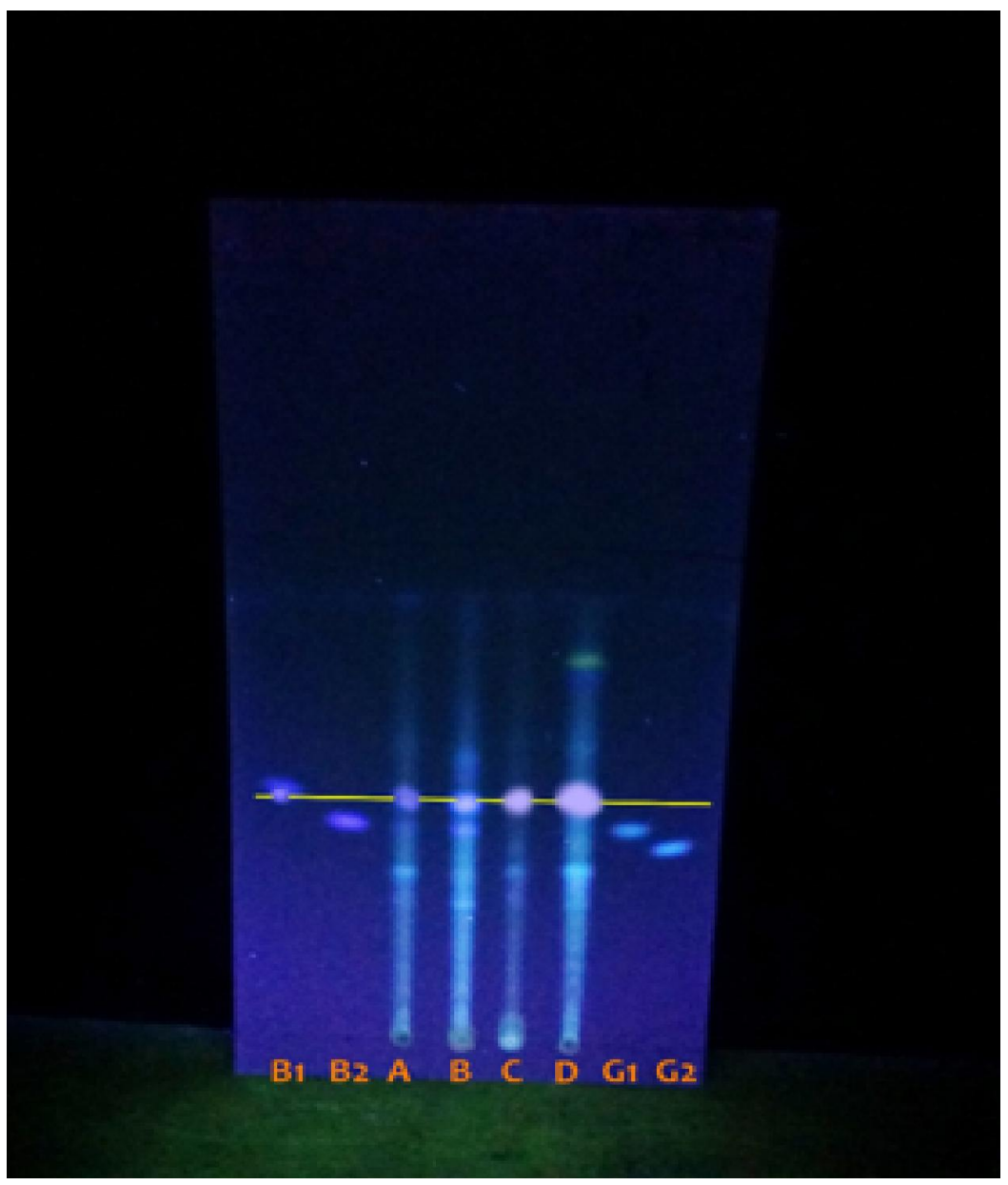

Figure 2 : Résultats de la CCM sur des pâtes d'arachide (blanc-matrice: A, B, C, D) contaminées à des concentrations connues à partir du standard AFB1 $(25 \mu \mathrm{g} / \mathrm{mL})$.

** A : $2 \mu \mathrm{g} / \mathrm{kg} ; \mathrm{B}: 5 \mu \mathrm{g} / \mathrm{kg} ; \mathrm{C}: 10 \mu \mathrm{g} / \mathrm{kg} ; \mathrm{D}: 20 \mu \mathrm{g} / \mathrm{kg}$

** Témoins : B1 (AFB1) ; B2 (AFB2) ; G1 (AFG1) ; G2 (AFG2)

\section{DISCUSSION}

Dans le but d'évaluer la limite de détection de l'AFB1 dans la pâte d'arachide, nous avons contaminé des échantillons «blanc-matrice» à des concentrations respectives de $2 \mu \mathrm{g} / \mathrm{kg}, 5 \mu \mathrm{g} / \mathrm{kg}, 10 \mu \mathrm{g} / \mathrm{kg}$, et $20 \mu \mathrm{g} / \mathrm{kg}$. Ces niveaux de contamination incluent les Limites Maximales de Résidus (LMR) admissibles dans le beurre de cacahuète ou la pâte d'arachide selon la réglementation de l'Union Européenne (2 $\mu \mathrm{g} / \mathrm{kg}$ ) et celle en vigueur au Nigéria (20 $\mu \mathrm{g} / \mathrm{kg}$ ). Le choix de contamination s'est porté sur l'AFB1 car c'est l'Aflatoxine la plus fréquente dans l'alimentation et aussi la plus toxique. Le métabolite de l'AFB1 libère l'AFM1 dans le lait. Son pouvoir cancérigène puissant et sa présence dans l'alimentation pour bébé ont contribué à instaurer des limites strictes (AFSSA, 2009).

Nos résultats démontrent que la méthode d'extraction et de détermination de l'AFB1 mise en œuvre permet de détecter des niveaux de contamination aussi faible que 2 $\mu \mathrm{g} / \mathrm{kg}$ dans la pâte d'arachide analysée. Cette valeur correspond bien aux limites de contamination autorisées pour l'AFB1 dans les arachides, les noix et les fruits séchés selon 
le règlement 1881/2006/CE qui impose des seuils de contamination inférieurs à 2,5 et 8 $\mu \mathrm{g} / \mathrm{kg}$ en fonction du produit et de son stade de transformation.

Nous n'avons pas testé la méthode pour des niveaux de contamination inférieurs à $2 \mu \mathrm{g} / \mathrm{kg}$. Toutefois, Tantaoui-Elaraki (1994), a pu démontrer au cours d'une expérimentation utilisant la même méthode que le niveau minimum de contamination de l'arachide avant l'extinction de la fluorescence était de $1,13 \mu \mathrm{g} / \mathrm{kg}$. Ces résultats viennent confirmer que la méthode utilisée permet de détecter des concentrations d'AFB1 correspondant aux limites maximales de résidus (LMR) autorisées selon la réglementation en vigueur au niveau régional et international.

Après avoir déterminé la sensibilité de notre méthode, nous l'avons appliquée dans le cadre de l'évaluation de la contamination par l'AFB1 des pâtes d'arachide vendues sur les trois principaux marchés d'Abidjan. Nous n'avons pas observé de contamination au-delà de $2 \mu \mathrm{g} / \mathrm{kg}$ dans l'ensemble des échantillons testés en duplicata. Cette absence de contamination soulève la question de la représentativité de l'échantillonnage réalisée au cours de notre étude. En effet, cette étude est préliminaire et avait pour but d'évaluer la contamination des pâtes d'arachides vendues sur les marchés d'Abidjan. Nous avons collecté les échantillons dans les 3 marchés les plus fréquentés de la Capitale et auprès des vendeuses les plus achalandées dans chaque marché. Nous estimons que les efforts d'échantillonnage réalisés permettent de dire que notre échantillon est assez représentatif de la pâte d'arachide consommée par la population abidjanaise. A titre de comparaison, dans l'étude de Sangaré-Tigori (2006), 10 échantillons d'arachide avaient été collectés sur les marchés de Treichville, Abobo et Adjamé.

Une des hypothèses pouvant expliquer l'absence de contamination des échantillons analysés serait le faible niveau de contamination des matières premières de fabrication de la pâte d'arachide, en l'occurrence l'arachide brute. En 2006,
Sangaré-Tigori a estimé que le niveau moyen de contamination des arachides vendues sur les marchés d'Abidjan par l'AFB1 était de 4,8 $\mu \mathrm{g} / \mathrm{kg}(1,5$ à $10 \mu \mathrm{g} / \mathrm{kg}, \mathrm{N}=10)$. Par contre, les études réalisées au Soudan par Jelinek (1999) et Creppy (2002) montrent des taux de contamination beaucoup plus importants dans les arachides prélevées sur les marchés locaux, soit $250 \mu \mathrm{g} / \mathrm{kg}$.

À la faible contamination initiale des arachides utilisées pour la fabrication des pâtes d'arachide, il faut prendre en compte le procédé de fabrication lui-même qui pourrait avoir un impact sur la réduction des taux d'Aflatoxines dans le produit fini (décontamination). Classiquement, le procédé de fabrication comprend le décorticage, le rôtissage, le blanchiment, le broyage, la stabilisation, le conditionnement et le repos (Knoden, 2003). Les travaux de Hussain et al. (2011) ont démontré que le rôtissage des arachides contaminées à $150{ }^{\circ} \mathrm{C}$ pendant 120 minutes entraînait une dégradation supérieure à $95 \%$ de l'AFB1 dans les arachides.

Nous n'avons pas de données sur les températures et durées de rôtissage appliquées par les fabricantes de pâte d'arachide. Toutefois, au vu de la faible contamination observée dans nos échantillons de pâte d'arachide nous formulons l'hypothèse que le rôtissage est réalisé à une température suffisante (probablement au-delà de $150{ }^{\circ} \mathrm{C}$ ) pour entraîner une décontamination de la pâte d'arachide. Bien entendu, cette hypothèse devra être vérifiée dans des études contrôlées futures qui devront prendre en compte les procédés de fabrication utilisés spécifiquement en Côte d'Ivoire.

Enfin, l'absence de contamination constaté dans nos échantillons, ne doit pas amener les autorités de contrôle des aliments, tel que le Laboratoire National de la Santé Publique, à relâcher leur vigilance. Notre méthode ne nous permettait pas de détecter des quantités d'Aflatoxine $\mathrm{B} 1$ inférieures à 2 $\mu \mathrm{g} / \mathrm{kg}$. Si ce seuil est parfaitement en accord avec les normes en vigueur pour l'AFB1 dans les arachides et dérivés, il faut considérer l'effet cumulatif des différentes Aflatoxines (multicontamination) qui peut entraîner des 
effets négatifs chez l'homme et particulièrement chez les sujets vulnérables comme les jeunes enfants. En conséquence, la CCM peut s'avérer très utile pour un dépistage préliminaire et un contrôle de la conformité des pâtes d'arachide relativement aux LMR admissibles pour les Aflatoxines. Cependant, lorsque la pâte d'arachide est destinée à l'alimentation de jeunes enfants il est indispensable de passer à des techniques d'analyses ayant des limites de détection plus basse de l'ordre de $0,1 \mu \mathrm{g} / \mathrm{kg}$ comme l'HPLCFLD ou l'HPLC-MS.

\section{Conclusion}

La CCM est une méthode simple, peu coûteuse, reproductible et fiable de surveillance de la contamination par les Aflatoxines des pâtes d'arachide vendues sur nos marchés locaux. Il s'agit d'une méthode semi-quantitative qui permet de discriminer les échantillons avec des taux de contamination en dessous ou au-dessus des LMR, avant une confirmation par HPLC pour les échantillons faiblement contaminés ou destinés à l'alimentation infantile. Dans le but de mieux éclairer notre connaissance, il conviendrait de conduire une étude expérimentale afin de vérifier que les conditions de fabrication de la pâte d'arachide en Côte d'Ivoire permettent une dégradation effective et efficace des A

\section{CONFLIT D'INTÉRÊTS}

Les auteurs déclarent n'avoir aucun conflit d'intérêts en rapport avec les travaux de cet article.

\section{CONTRIBUTIONS DES AUTEURS}

$\mathrm{AD}$ a contribué à l'élaboration $\mathrm{du}$ protocole de recherche, à l'interprétation des résultats et à la rédaction du manuscrit. MGBI a contribué à la validation du protocole de recherche, à l'interprétation des résultats et à la révision du manuscrit. DKN a réalisé l'échantillonnage sur le terrain, les analyses des échantillons et a contribué à la révision du manuscrit. JAY a supervisé les travaux et a participé à la révision du manuscrit.

\section{REMERCIEMENTS}

Les auteurs remercient le Laboratoire National de la Santé Publique de Côte d'Ivoire (LNSP-ci), Structure au sein de laquelle les travaux de recherche ont été menés, et qui a mis gracieusement à la disposition des chercheurs le matériel, les réactifs et l'assistance technique.

\section{RÉFÉRENCES}

Abdellah Z. 2004. Détermination des mycotoxines dans les aliments et étude de la réduction des Aflatoxines par les bactéries lactiques isolées des ferments panaires traditionnels. Thèse de Doctorat, Université Sidi Mohammed Ben Abdellah, Fès, $\mathrm{N}^{\mathrm{o}}$ 46, p. 162.

AFSSA. 2009. Évaluation des risques liés à la présence de mycotoxines dans les chaînes alimentaires humaine et animale, Rapport final Mars 2009, 44.

Betina, V. 1989. Mycotoxins: Chemical, biological and environmental aspects. In Bioactive Molecules, Betina V (ed.). Elsevier Applied Science : London: 114150.

Boutibonnes P, Jacquet J, Teherani A. 1969. Chromatographie rapide en couche mince des flavotoxines dans les aliments. Bull. Acad. Vét., 42 : 825-833.

Costanzo P, Santini A, Fattore L, Novellino E, Ritieni A. 2015. Toxicity of aflatoxin B1 towards the vitamin D receptor (VDR). Food Chem. Toxicol. 76 (2): 77-9. DOI: http://dx.doi.org/10.1016/j.fct.2014.11.0 25

Creppy E. 2002. Update of survey, regulation and toxic effects of mycotoxins in Europe. Toxicol. Lett., 127 (1-3): 19-28. DOI: $\quad$ https://doi.org/10.1016/S03784274(01)00479-9

Hussain A, Ali J, Akhter S, Shafqatullah. 2011. Degradation of aflatoxins by roasting in contaminated peanuts. Pak. $J$. Biochem. Mol. Biol., 44 (2): 56-59.

IARC. 2002. Some traditional herbal 
medicines, some mycotoxins, naphthalene and styrene. IARC Monogr Eval Carcinog Risks Hum., 82: 1-556.

INRS. 2009. Documents pour le Médecin du Travail $\mathrm{N}^{\circ} 119$, 3e trimester, 304.

Institut National de Statistiques de Côte d'Ivoire (INS). 2014. Rapport : Recensement Général de la Population et de l'Habitat 2014. Disponible sur www.ins.ci/n/documents/RGPH2014_ex po_dg.pdf

Jelinek F.C, Pohland A.E, Wood G.E. 1989. Review of mycotoxin contamination. Worldwide occurrence of mycotoxins in foods and feeds an update. J. Assoc. Off. Anal. Chem., 72 : 223-230.

Knoden J-L, Dufour L, Bindelle J. Fabrication $\mathrm{du}$ beurre de cacahuette. Collection "Manuels Techniques". Ed. Ingénieurs Sans Frontière (ISF). Bruxelle, Belgique. Disponible sur internet, consulté le 15 mai 2016.

Sangare-Tigori B, Moukha S, Kouadio J, Betbeder A-M, Dano S, Creppy E. 2006. Co-Occurrence of Aflatoxin B1, Fumonisin B1, Ochratoxin A and Zearalenone in cereals and peanuts in Cote d'Ivoire. Food Additives and Contaminants, 23(10): 1000-1007. DOI: http://dx.doi.org/10.1080/026520305004 15686

Tantaoui-Elaraki A, Benabdellah L, Majdi M, Elalaoui MR, Dahmani A. 1994. Recherche de mycotoxines dans les denrées alimentaires distribuées au Maroc. Actes Inst. Agron. Vet. (Maroc), 14 (3): 11-16.

Wacoo AP, Wendiro D, Vuzi PC, Hawumba JF. 2014. Methods for Detection of Aflatoxins in Agricultural Food Crops. $J$. Appl. Chem., 2014: 11-15. DOI: http://dx.doi.org/10.1155/2014/706291 